\title{
The research of evaluation method of ATM shifting quality based on
}

\author{
neural network
}

\author{
Cheng Wenming
}

\author{
Automotive Engineering institute, Jiangxi University of Technology, Nanchang 330098, China
}

Key words: ATM shifting quality; Evaluation method; Neural network;

\begin{abstract}
With the development of the modern automobile industry and the continuous improvement of overall performance of the vehicle, the contents of shifting quality grow increasingly richer. The research and solution of the improvement of shifting quality just from the perspective of ride comfort can not adapt to the requirements of improving the general proficiency of shifting quality comprehensively. The contents of shifting quality of modern significance include: ride comfort index such as shifting impact caused by the change of transmission ratio, speed fluctuation and vibration noise; the shifting fuel economy and emission index considering the energy and environmental issues; the lifespan index of components in the power transmission system affected by shifting peak load; shifting quality directly affects the vehicle's power, economy, ride comfort, safety, driving control and the lifespan of power transmission system. With more and more in-depth research of automatic transmission theory and people's requirements of the shifting quality, the study on rational evaluation index for shifting quality and scientific evaluation method is of great significance, laying theoretical foundation for the construction of dynamic comprehensive evaluation system of the shifting quality and the research for improving the quality. The establishment of a model of objective evaluation method for shifting quality based on neural network is proposed. It can realize the carrier to achieve the objective evaluation and develop evaluation software.
\end{abstract}

\section{Introduction}

Aiming to the four multi-aspect and complicated indexes above of the shifting quality, how to determine the duty parameters which can both reflect truly whether each index is good and be measured easily and their respective physical meaning and mathematical expressions is the most important problem faced by the research of the evaluation system of shifting quality. In view of the complexity of the relationship between each index, analysis of each influence factor and its relationship with each other is the basis for the establishment of comprehensive evaluation system. The aims of studying the evaluation system of shifting quality are to study the requirements of different intentions to each index, determine the weight allocation scheme of each index under different working conditions and establish a dynamic integrated system which can reflect shifting comprehensively and truly.

The study on shift quality evaluation includes two aspects: to determine the evaluation index and scientific evaluation method. The focus of the evaluation is to ensure the connection between human as objective evaluation index and his subjective feeling and to observe its relevance and consistency. In order to obtain the subjective evaluation value of shifting quality from the objective data in a shifting event, the following techniques can be adopted. 
Technique 1: fitted equation (use the analysis and evaluation of the vehicle longitudinal acceleration)

Experiments show that this method can only be used in low-gear cases. The dashed line is the ideal shifting curve. The deviation from the ideal curve means that shifting quality evaluation rank will fall. The curve in the figure is divided into 5 parts: (1)linear; (2)exponential decay; (3)linear; (4)the index increased; (5)twice oscillation curve. The A area is speed. The smaller A+ B area is, the smaller the energy change will be. Because the shake of the driver in the seat is caused by the change of acceleration, the weight of the B area is larger than the weight of A. This method is only for a vehicle whose correlation is calibrated, but it cannot be used on different vehicle universally, so it must be recalibrated for the evaluation of other vehicles.

Technique 2: neural network identification technology

In order to better evaluate all shifting situations, nonlinear neural network technology can be utilized. The application of artificial intelligence tools is to solve the basic theory of the consistency between subjective and objective evaluation criteria, such as genetic algorithm, neural network, vague logic, prediction algorithm, etc. The paper studies the shifting quality evaluation based on this method.

Because different drivers have different driving experience, driving habits and driving intentions, such a subjective evaluation method is too subjective. The result of its application is to extend the system's development cycle and increase development costs.

\section{The analysis of AMT shifting process and the determination of evaluation index}

To evaluate AMT shifting quality, an equivalent mechanical model of the power transmission system should be constructed. By modeling, the connection between each key parameter can be fully understood in the entire process of shifting to formulate index for the analysis of shifting quality's influence factors.

The clutch needs to coordinate with engine throttle and shifting operation. The control has a great influence on the normal operation of AMT. In order to improve the shifting quality of AMT, a model where the power train can be simplified to the both ends of the clutch in the process of clutch engagement should be constructed. Its main features are grasped to analyze the process of clutch engagement.

The clutch's starting or shifting can be simplified as the following mathematical model. (Fig .1)

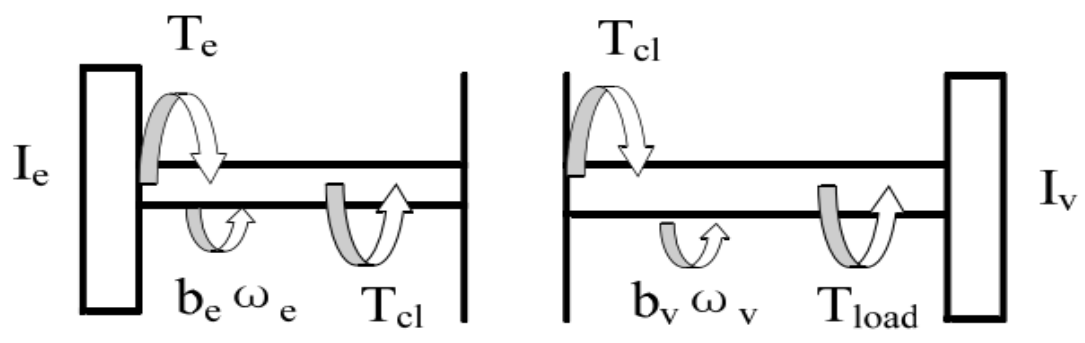

Figure. 1 The simplified mathematical model for the process of clutch engagement

In the process of shifting, here are the influence factors for the quality:

When shifting, the torque eT from the engine is uncoordinated with the resistance torque rT from the wheel;

The engine's speed changes; 
There is a relatively large speed difference between the active and passive gear when they are synchronous;

After shifting up there is a speed difference between the active and passive driving plates when the clutch engages.

The length of AMT shifting time is determined by the speed of the separation and combination of the clutch. The combination will not only affect the ride comfort of the shifting but also affects the service life of the clutch. Comfort is a subjective thing. Different drivers feel differently. But in general, the smoother the change of vehicle acceleration is, the smaller the peak of impact degree will be, and the more comfortable the shifting will be. Thus, the impact degree can be used as the index to evaluate drivers' ride comfort.

The impact degree is expressed as the change rate of the vehicle's acceleration a. Here is the formula.

$$
j=\frac{d a}{d t}=\frac{r_{w}}{i_{0} i_{g}} \frac{d^{2} w_{v}}{d t^{2}}
$$

Nowadays, the degree of impact attracts more and more attention in the field of vehicle design and control, widely accepted as an important indicator of shifting quality, especially in the design of AMT where the researchers usually use the maximum impact degree to evaluate the performance of the control strategy. The smaller the impact is, the better the performance will be. If the impact is greater than $10 \mathrm{~m} / \mathrm{s} 3$, the control strategy needs to be modified.

Shifting time $(\mathrm{T})$

Because the clutch separation does damage to the power performance of vehicle, if in the whole process of shifting the power is interrupted for too long, the accelerating performance of the vehicle will be affected as well as the ride comfort. Hence, shifting time should be as little as possible. The less the shifting costs, the shorter the power interruption will be and the better the power performance and the shifting quality will be. From the statistics, AMT shifting time is $800 \mathrm{~ms}$ higher than that of the traditional AT, which reflects one reason why AT shifting quality is better than other transmissions.

Solution to the shifting time:

The shifting process includes clutch separation, clutch engagement and shifting operation. The shifting time refers to the time when the clutch begins to separate and then engages fully. It includes: (1) the time of the clutch separation, (2) the time of plucking former gear (3) the time of selection (4) the time for synchronization and engaging new gear (5) the time for reengaging the clutch.

The speed fluctuation quantity reflects the engine control quality in the shifting process, represented by the speed difference of the engine and the input shaft. When shifting, speed fluctuation of the engine will cause the engine to roar. This factor should not be ignored in the subjective evaluation, which can be understood as the noise of shifting. If the amount of fluctuation is smaller, the quality of engine control will be better. In the shifting process the control of the target engine speed should meet:

$$
w_{e}^{t}=\frac{i_{g(n+1)}}{i_{g(n)}} w_{e}
$$




\section{The theoretical basis of neural network}

The full name of neural network is Artificial Neural Network, ANN, which is a hotspot of modern high-tech. It is a product of mutual cross, mutual penetration and mutual promotion of life science and engineering. Due to its advantages such as highly nonlinear mapping, it is extensively applied in many fields such as pattern recognition, image processing, signal processing, automatic control, system identification, etc. The artificial neural network studies human's intelligent behavior and simulates the human brain to process information according to the physiological structure of the human brain. As neuron is the basic unit of brain tissue, artificial neural network is a basic processing unit.

Currently, there are dozens of kinds of neural network models, but the most extensively used one is BP neural network (Backpropagation Neural Network). The following is a brief introduction of the structure and algorithm of BP network.

\section{The structure of BP network}

BP network is a neural network of three or more layers, including input layer, middle layer (hidden layer) and output layer. There is a connection between upper and lower layers, while between each layer of neurons exists no connection. When a learning sample is provided to the network, neuronal activation values are communicated to the output layer from the input layer through the middle layer. Each neuron on the output layer obtains the network input response. Next, according to the direction of reducing target output and actual error, the connected weights are modified from the output layer through each middle layer and finally back to the input layer. This algorithm is called "error back-propagation algorithm", namely, BP algorithm.

The structure of BP net is shown in Fig .2. In addition to the input and the output nodes, the network has one or more layers of hidden layer nodes. There is no coupling of the nodes in the same layer. The input signal is transmitted from the input layer nodes through the hidden layer nodes by turns and then back to the output node. The output of each node only affects the output of the next node. Each node has a single neuronal structure shown in Fig .2, whose unit feature (transfer function) is usually the Sigmoid function shown in Fig .3, but the unit characteristic of the node in the output layer is linear sometimes.

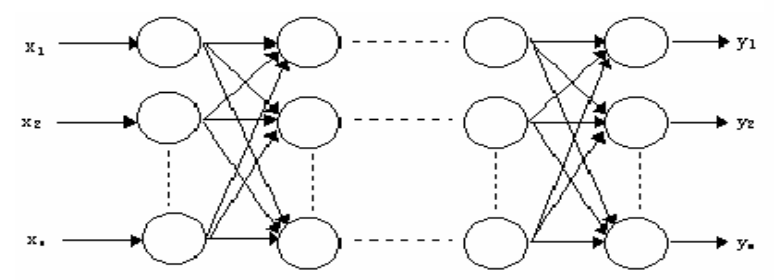

Figure. 2 The structure of BP network

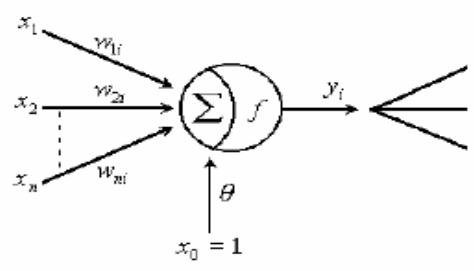

Figure. 3 the model of neuron structure 
The artificial neuron is the basic processing unit of the neural network. It is to simplify and simulate biological neurons. Fig .4-2 shows a simplified neuron structure, which is a multi-input and single-output nonlinear element. The relationship of the input and output can be described as:

$$
\begin{gathered}
I_{i}=\sum_{j=1}^{n} w_{j i} x_{j}-\theta_{i} \\
y_{i}=f\left(I_{i}\right)
\end{gathered}
$$

In system modeling, identification and prediction, as for the linear system, in the frequency domain, the transfer function matrix can express very well the black box input / output model of the system; in the time domain, the input and output in the system can also be described by using the autoregressive moving average (ARMA) estimation method through various model parameters. As a result, the prediction problem of linear system is relatively perfectly solved. However, the nonlinear system generally adopts the model based on the nonlinear autoregressive moving average (NAMA) to predict, but it is difficult to find an appropriate parameter estimation method for this kind of model. Therefore, there are great difficulties in the identification of traditional nonlinear systems both in the theory research and practical application.

\section{Conclusion}

On the vehicles equipped with a modern level of automatic transmission, because there are effective working range of engine in output torque and speed, the shifting will cause fluctuation of the vehicle speed and engine speed, making the passengers uncomfortable. The study on evaluation method of shifting quality is of great significance to improve shifting quality.

Take the AMT model as the research object. Large numbers of samples from the test data, including the key indicator parameters in the shifting process and the corresponding subjective evaluation of shifting given by the driver through scoring, are considered as training samples and test samples of neural network. After the network is trained and testing is completed, build an objective evaluation model for shifting quality in the Matlab/Simulink environment. Finally, develop objective assessment software for shifting quality based on the Matlab/GUI. The comparative analysis of network computing results and the subjective evaluation results confirm the feasibility of the method.

The AMT evaluation method for shifting quality based on artificial neural networks has broad prospects for development, but in the course of the study the following aspects should be researched more comprehensively and deeply.

How to realize real-time online. This paper makes a theoretical preparation for the future development of neural network with online learning function;

According to different models, different objective evaluation emphasizes different evaluation criteria. The evaluation index can be obtained or abandoned according to the needs. Train different network, so as to construct a dynamic integrated evaluation system of shifting quality.

Driver's intention identification. The randomness and uncertainty of driving intentions as well as the limitation of available parameters bring difficulties to identify the driving purpose. How to accurately identify the driving intentions and classify reasonably the working conditions is an indispensable part of the dynamic integrated evaluation system under different working conditions. 


\section{Acknowledgements}

This work was financially supported by the key subject building project (vehicle engineering) of Jiangxi University of Technology.

\section{References}

[1] Ngo N H, Landsheere X, Pangui E, et al. Self-broadening and-shifting of very intense lines of the $1 \leftarrow 0$ band of $12 \mathrm{C} 16 \mathrm{O}[\mathrm{J}]$. Journal of Quantitative Spectroscopy and Radiative Transfer, 2014, 149: $285-290$.

[2] Sharma S, Singh V K, Kishore J, et al. A Study on Customer Perception towards Service Quality and Delivery with Reference to E-Banking[J]. Available at SSRN 2392234, 2014.

[3] Koteswari S, Paul P J, Indrani S. VC of IRIS Images for ATM Banking[J]. International Journal of Computer Applications, 2012, 48(18): 1-5.

[4] Koteswari S, Paul P J, Indrani S. VC of IRIS Images for ATM Banking[J]. International Journal of Computer Applications, 2012, 48(18): 1-5.

[5] Sharma N, Singh A, Dhyani R, et al. Atm spheric Pollution Research[J]. 2014.

[6] Bickford E, Holloway T, Karambelas A, et al. Emissions and Air Quality Impacts of Truck-to-Rail Freight Modal Shifts in the Midwestern United States[J]. Environmental science \& technology, 2013, 48(1): 446-454.

[7] Ying H E. Research of the AMT vehicle on starting process[J]. Machinery Design \& Manufacture, 2011, 5: 069.

[8] Petrova T M, Solodov A M, Solodov A A, et al. Measurements of O 2-broadening and-shifting parameters of water vapor spectral lines in the second hexad region[J]. Journal of Quantitative Spectroscopy and Radiative Transfer, 2011, 112(18): 2741-2749.

[9] Baki A T M. Socio-economic impacts of Gorai riverbank erosion on people: a case study of Kumarkhali, Kushtia[D]. BRAC University, 2014.

[10]Asfin R E, Domanskaya A V, Maul C. Broadening and shifting coefficients of rotation-vibrational lines in the fundamental and first overtone bands of $\mathrm{HCl}$ and $\mathrm{HBr}$ induced by oxygen and air[J]. Journal of Quantitative Spectroscopy and Radiative Transfer, 2013, 130: 296-303. 